\title{
Lin Fengmian's Portrait of a Lady: The Influence of Western Art and the Reformation of Chinese Tradition
}

\author{
Enyu Cui*
}

\author{
AP Program, Guangdong Experimental High School, Guangzhou, Guangdong, China \\ ${ }^{*}$ Corresponding author. Email: 20140543@stu.nun.edu.cn
}

\begin{abstract}
From the late 19th century to the early 20th century, many artists appeared along with Shanghai. Some learned paintings in China while were studying abroad, like in Paris or Japan. All these artists want to mix Chinese traditional objects with Western painting techniques well. By using a bright color, Lin Fengmian might be the representative artist of it. Later in his life, he was more willing to paint the Portrait of the Woman, and also, there are rarely some findings of the subject matter in the image. Many other objects can be painted, like landscape or still-life. It is deserved to discuss why he chose to paint the Portrait of the Lady. To begin with, for the landscape paintings, the objects in the picture, like mountains, trees, and tiny houses, are not close enough to investigate, while the lady sits face to face with the audience. Also, it was just using the western drawing techniques Lin learned from France to build up the scenario, which is very common to see in traditional Chinese paintings. Little distance between the observer and the object can focus on the light, texture of clothing, and hairstyle. A closer look, audiences can recognize the influence of the different schools of painting from his description. This article will get a closer look at the picture to get a complete idea about the objects in it and the influence he accepted, even about the reformation of the Chinese drawing style.
\end{abstract}

Keywords: Lin Fengmian, The Portrait of a Lady, drawing style.

\section{INTRODUCTION}

One of the critical assumptions of the qualitative method is that brushstrokes constantly coordinate the relationship between the shape, color, and space in the picture [1]. Thus, observing through the image of Lin FengMian, the emotions and ideas that he wanted to convey could be easily detected by the audience.

His paintings were destroyed by himself mostly during 1966-1976, the Cultural Revolution. Therefore, people can rarely watch the real version of the Portrait of Lady and know the exact time he created it. Thus, we can only observe the picture downloaded from the Internet and try to find the one with precise time. The exposure to light might affect the color, though. Therefore, it will seem like one of the works already auctioned at a specific time, created from the late 1950s to the early 1960s. Some artists who studied abroad during the same period, like $\mathrm{Xu}$ Beihong, also played a crucial role in the revolution of the art style in China. He tried to mix Western and Eastern art. However, their fusion focused on drawing techniques instead of color, objects, and ideas. The Running Horse, for instance, won lots of reputation for him. He utilizes perspective, spatial, light, shadow, water, and ink to form a unique ink effect. However, there are still some differences between him and Lin Fengmian, which will be argued in the following article by pointing out four aspects.

\section{INFLUENTIAL ART SCHOOLS IN LIN'S PAINTINGS}

For the whole scenario, the appearance of Impressionism, Expressionism, and Fauvism is strongly approved. The woman's characteristics allow people to remind the painting, Portrait of Madame Matisse, created by the representative artist of Fauvism: Henri Matisse. Although the facial features are similar to Matisse's painting style, the color he used and the emotions he wanted to convey are different from Matisse. For the coincidence that clothing colors are the same as flower colors, the woman's elongated neck is also similar to the form of a vase. People can imagine that maybe the vase, the flowers, and the woman are mirrored. People would like to regard this abstract idea as a part of Expressionism. Besides, he applies the light ingeniously by reflecting it on the texture and color lump of clothing. The sleeves are 
pertinent to the background; both are dark, while the clothes worn inside are bright enough to form the comparison, just like the background.

From the comparison between landscape painting and a portrait of ladies, there are some traces of using Impressionistic techniques by utilizing light on color and the brushwork. The article takes the painting Qiuyan as an example. In the painting, the stippling, the typical brushwork of Impressionism, instead of water and ink. The texture of the clothing is similar to the one of the curtains. The Curtain, which seems like the window's screen, is permeable enough for light to pass through and reflect on the figure. The light passes through the curtain, reflecting on the clothing of the lady's legs. The brightness of the ribbon ties on the clothing inside also indicates a light behind the lady. Obviously, the lady's body leans to the left, and she also put her left hand up, then the left side ribbon is darker than the right side. Above all, these characteristics are show us to effect of Impressionism on the painter. Similarly, the landscape painting also applies the stippling brushstrokes and his colors to cover black, white, and other bright colors. The tints let people recognize that the idea he wants to fuse traditional Chinese painting and Western painting. The audience can trace this movement back to the experience of studying abroad in France. In contrast, the brightness of colors conveys different kinds of feelings to the audience. Pigments apply in the portrait of the lady are also bright, though. It provides an atmosphere of quietness, like gray and dark blue. The colors in still-life paintings, like gold and light red, enable people to remind some strong and joyful feelings.

The uncertain emotions transmitted by her eyes and mouth make the image looks filmier, which is the most knowable distinction of Impressionism. The square composition used by Lin FengMian is in accord with the focus perspective in Western drawings. It benefits some close-up depictions. This composition allows the audience to focus on the woman itself directly. For the inspiration of Matisse, he likes to use some lines to describe the figure's clothing, face, even the background. The gray background builds up a strong contrast with the precise figure. Using light to emphasize the woman in the middle. In the drawing, the figure stands in the middle, bright; both left and right sides are in the dark. Therefore, the woman set in the middle can bring all the attention.

Late in his life, most of his paintings cover the dark color or the color with low saturation. It is worthy of discussing why Lin transforms the color so suddenly. From his autobiography, he went through a difficult time from 1938 to 1977. During that time, there was an important political event took place in China, the Culture Revolution. At that time, he has nothing to do but destroy all his paintings to seek survival. Moreover, because of his arguments which are against the government, he was highly criticized. None of them can understand his ideas.
Under that situation, he felt such loneliness, which led to the dark color in his paintings.

\section{THE FUSION}

Lin was capable of painting several kinds of painting, even water and ink painting. Portrait of Lady is painted in several artistic forms, like oil painting and water and ink painting. Although the subject matters are similar, there are still quite some differences between them. Firstly, the texture of clothing in water and ink painting is solid, while the oil painting can make people dynamic. In oil painting, the light would able to be figured out through the light and shade contrast. By contrast, the image needs to leave some white spaces between the subject matter, like the lady's arms, so that the audience would realize the existence of light behind the lady.

Additionally, in the painting of water and ink, he leaves the traces of ink consciously. The watcher can easily recognize this is traditional water and ink painting through the unequivocal brushstrokes. At a closer look, the observer even can find out there is still some water remaining on it. However, people can merely see any signs of brushwork in oil painting.

The fusion of traditional Chinese painting and Western painting reflects the color and the flow of lines. The lady puts her hair into two circles, just like the ribbon and her legs. She crosses her legs in the same way as tying the ribbons. Coiling lines into circles and curve lines makes this pattern more likely to calligraphy, a kind of traditional Chinese handwriting form. There are also several forms of calligraphy, like writing straight or composing Hanzi with curved lines, for instance, cursive scripts and regular scripts. No matter the utilization of colors or the curved lines, both are evidence of Lin trying to mix Eastern and Western art.

People can quickly remind the Dunhuang fresco of the clothes the woman wears and the way she sits. The clothing she wears is so light that it flows down to the floor. With wind, it can flow up. Her head is lean to the left side, and she also holds the instrument, which is lean to the right side. This kind of balance or position can be easily seen from the women who painted on the frescoes. Take the picture from Cave 407 of Mogao Grottoes in Dunhuang. The way it sits and positions its points are so similar to the Portrait of Woman. The most typical images of the frescoes are the flying apsaras. From the Sixteen Kingdom to Tang Dynasty, Dunhuang apsaras rose and flourished and brought up the dancing art. The women who earn alive by playing an instrument are called Yuechi. The magnificence of dancing art at that time can be adequately illustrated by the grand scale of the scenes painted in Dunhuang frescos. One-third of Dunhuang apsaras are flying withheld an instrument. The forms and gestures of Yuechi are the same as those of various musical instruments played by Yuechi in past 
dynasties. However, they are only decorated with flying ribbons and painted with passing clouds and flowers. Researchers point out that most of the shapes and posture of apsaras borrowed and imitated from acrobatics, dancers, martial or sports [2-4]. Thus, Lin Fengmian depicting the woman by consulting from apsaras is best to show Western technique and Eastern culture.

\section{RELATION TO CHINESE TRADITION}

At first glance, the woman is sitting quietly and elegantly, and her eyelids drooped with a slight nod. The woman playing a stringed instrument came from ancient China, called Ruan. A pair of slender eyes are depicted by two lance-shaped black lines, which fit with the nose lines. The painter used two lines to connect her nose and eyebrows, making them look thin. Her lips consist of two lines, and they are separate. Both sides of the end of the upper one let the woman seems like smiling, but not actually. This kind of drawing technique is always used by drawing her eyes and eyebrows. It enables the image of the woman to be covered by a layer of uncertainty. All these facial features are similar to the traditional Chinese painting, called Portrait of Flower-wearing women, created by Zhou fang. Similarity indicates the idea Lin FengMian wanted to convey is close to traditional images. Portrait of Flower-wearing women describes there are five women in gorgeous clothing taking a walk in the garden. It is separated into four parts: gathering flowers, appreciating flowers, having a walk, and playing with pets. All the subject matter uses lines to paint the body shape rounded. He let the clothing seem so light to swing when the wind blows and thin enough so that the audience can see through it to the skin of the five women. The five women are all dressed in ornate dressing: color, the faint color of faith, and clothes decoration. The resemble of Portrait of a lady between his and Zhou Fang is remarkable. The women in both paintings have elongated fingers. Combining all the features allow people reminded Lin DaiYu quickly, who is the representative character of weakness, an elegant woman.

In the novel The Dream of Red Mansion, there is some specific description about Lin DaiYu: Her dusky arched eyebrows were knitted and yet not frowning. Her speaking eyes held both merriment and sorrow; her very frailty had charm. Her eyes sparkled with tears; her breath was soft and faint. In repose, she was like a lovely flower mirrored in the water; in motion, a pliant willow swaying in the wind. She looked more sensitive than $\mathrm{Bi}$ Gan, more delicate than Xi Shi. The Dream of Red Mansion is one of the well-known novels in China; rarely have people not read through the book. The weak image of Lin DaiYu is so impressive that she unconsciously thinks of her once mentioned weak lady. She then became the representative character of weakness. It is a kind of stereotype of a woman in ancient China. People thought the women in a tall and low-weight are always the weakest ones. This description highly matches with the lady in the painting.

The image in the drawings wears the Ming Dynasty or the Song Dynasty, pulling the hair back into a bun. The clothing is big enough to hide the distinction of a lady's body shape, rarely working out if the woman sits up straight or sits freely. Curved lines created by the clothes are opposite to the size of the painting, which is squaremeaning of lines is always related to the word-tough. On the contrary, the idea Lin FengMian wants to transmit is not tough at all. The vase put behind her is one of the figurative vases in the Song Dynasty. It seems to combine the characteristic of both the olive-shape vase and Straight-neck bottle. From a closer look, it is easy to find out that the clothing colors are also related to Song Dynasty. Song Dynasty was famous for its permeability colors. Flowers are put in a way that doesn't fit the vase period: Song Dynasty desires to put flowers simply $[5,6]$. Literati of the Song Dynasty would like to put one branch and a flower into the vase, while many flowers are placed in the vase in that painting.

\section{THE AMBIGUITY}

More and more artists were studying abroad. They tried to reform traditional Chinese paintings, like Lin Fengmian, Xu Beihong, San Yu, and Pan Yuliang. First, Lin Fengmian wanted to introduce the spirit of creating the works into China, and then he mixed the Western drawing technique or oil paintings with the elements of Chinese, like Ruan [7]. On the other hand, Xu Beihong used sketch techniques to improve literati [8]. However, they separate into two different kinds of groups: realism and modernism. Therefore, it is worthy of discussing which played an essential role in reforming the painting style.

For modernism, Lin Fengmian and San Yu both have studied in Paris. They are more willing to paint the picture with some lines, which Matisse mainly influences. San Yu's paintings' lines are so easy that people can realize that he is a free-easy person and eager to get freedom [9]. Their subject matters are quite different, though. San Yu prefers to draw some naked women, while Lin wants to paint the women who act like the Dunhuang apsaras. It is difficult for the audience at that time to accept naked women as the subject matter. Because of his pureness, he wants to record all the beautiful things in the world by drawing pictures of them, like flowers, animals. But, of course, it also included the women. He paints naked women without any eroticism but full of innocence. The easy lines can strengthen this feeling. Take horse as an example. Compared with the horses painted by Xu Beihong, his horses cover in many bright colors instead of black and white. Without drawing details, he is more willing to paint it more personalized and abstract, which is quite different from the horses decided by Xu Beihong. At the period realism flourished 
in China, modernism was entirely unacceptable, which caused lots of controversies.

As for realism, $\mathrm{Xu}$ Beihong and Pan Yuliang are the representative artists of it. The reason why Xu Beihong prefers realism is entirely like Pan Yuliang. Since his childhood, he constantly reminds the reality, making it easier for him to empathize with realistic paintings which reflect social reality. Also, this experience makes him a responsible artist, leading him to reform the Chinese traditional painting style as the main work of his life. From his painting, rarely can find out the influence of Impressionism or other school culture. He is famous for depicting horses in detail. Pan Yuliang studied abroad when modernism was in vogue in Europe; she researched academism rather than modernism. She looked about the human, like anatomy, at first. Then she learned the stipple from Impressionism and colorful images from Fauvism. At that time, her works had a solid foundation of realism and had changeable and charming expression techniques of light. The picture is concise, and the composition is complete and rigorous. After she came back to China, she tried to use water-ink to paint. From the influence of Liu Haisu, she attempted to blend Western art and Eastern art [10]. For example, she used brush ink to draw Western body paintings on rice paper and the materials of color ink to integrate the techniques of lines. She started to draw from drawing the women. Constrained by the feudal ideology and social environment, the subject was minimal at the beginning. But she still consisted of painting it in a dilemma. It is challenging to define innovation precisely. However, without these difficulties, people cannot understand the background and the works behind these artists, like politics. Therefore, for the researchers who want to study more profound about the artists, this ambiguity might be the best way to go through it [11].

\section{CONCLUSION}

This paper examines the subject matter and the specific position of the woman painted by Lin Fengmian. Thus, people can get a more comprehensive understanding of his painting, Portrait of the Woman. The article has significantly influenced Expressionism, Impressionism, and Fauvism by observing the lines, style, color, and composition. The influence is only pointed out from the drawing technique; the objects he painted always focus on the Chinese traditional culture, like the vase, the similarity between the woman and the apsaras. Besides, these can be also seemed like a blend of Western and Eastern. Choosing the observed object only by locating the time is not strong enough to convince people. Several dimensions of the educated ideas about fusing Eastern and Western painting help select the Portrait of Lady as the subject matter. From this passage, the concrete examination of Lin Fengmian's paintings has to be approved well, which rarely studies investigate.
Compared with other artists, Lin has little renown. As more and more get close to his ideas or works, people can remind him quickly when discussing the Shanghai School Culture.

\section{REFERENCES}

[1] Ma Zhongbiao. A study on the Expression of brushstrokes in post-Impressionist oil paintings. Diss. Yunnan Normal University.

[2] Xie Shengbao. "The source of the shape and posture of Dunhuang Apsaras." Dunhuang Research (2001).

[3] ŚLĄCZKA, ANNA A. "Temples, Inscriptions and Misconceptions: Charles-Louis Fábri and the Khajuraho "Apsaras." The Rijksmuseum Bulletin 60, no. 3 (2012): 212-33.

[4] Roy, Oly. "THE OTHER WOMEN - HEAVENLY AND EARTHLY: NOTES ON 'APSARĀS AND VEŚYĀS."' Proceedings of the Indian History Congress 75 (2014): 186-93.

[5] Zhishui, Yang. "Song Dynasty Vases (heaping)." Palace Museum Journal (2007).

[6] Rawson, Jessica. "Ornament as System: Chinese Bird-and-Flower Design." The Burlington Magazine 148, no. 1239 (2006): 380-89.

[7] Mingxia, Cheng. "On Theoretical Value and Meaning of Comparing Study on Xu Beihong and Lin Fengmian." Journal of Nanjing Arts Institute (Fine Arts \& Design) (2011).

[8] Zhang Guangcai. "On Xv Beihong's sketch view." Art Observatory 11 (2006): 14-15

[9] Peng Yuanyuan. "Analysis of The Simplicity of Sanyu's Painting Language." Collection 27 (2019).

[10] Yan-li, S. U. N. "A Combination of Chinese and Western Painting__ A Course of Pan Yuliang's Art of Paintings." Journal of West Anhui University (2009): 04

[11] Rule, Ted. "Pan Yuliang, Xu Beihong and the revolution in Chinese art." Quadrant 64.4 (2020): 100-105. 